\title{
Analysis and Optimization of Asymmetrical Double-Sided Electrodynamic Suspension Devices
}

\author{
Jiaheng Duan ${ }^{\circledR 1,2}$, Song Xiao ${ }^{(1,2}$, Kunlun Zhang ${ }^{1,2}$, Mihai Rotaru ${ }^{3}$, and J. K. Sykulski ${ }^{\circledR 3}$, Fellow, IEEE \\ ${ }^{1}$ Key Laboratory of Magnetic Suspension Technology and Maglev Vehicle, Ministry of Education, Chengdu 610031, China \\ ${ }^{2}$ School of Electrical Engineering, Southwest Jiaotong University, Chengdu 610031, China \\ ${ }^{3}$ Electronics and Computer Science, University of Southampton, Southampton SO17 1BJ, U.K.
}

A novel electrodynamic suspension device using an asymmetrical double-sided Halbach array is proposed in this paper. A 3-D analytical model describing the eddy current field and the electromagnetic force is established via the second-order vector potential formulation. The validity of the analytical model is verified by both finite-element simulations and an experiment. The configuration of the proposed suspension device is optimized exploiting a dynamic kriging surrogate model assisted by a multiobjective genetic algorithm. The multi-objective optimization is performed utilizing the 3-D analytical model.

Index Terms-Electrodynamic suspension (EDS) device, kriging surrogate model, multi-objective optimization, null flux.

\section{INTRODUCTION}

$\mathbf{T}$ HE electrodynamic suspension (EDS) system that consists of a linear Halbach permanent magnet (PM) array and conductive plate is attractive for maglev trains due to its simplicity and reliability. So far, a single-sided structure has been implemented on the prototype of Magplane vehicles [1]. However, the excessive drag force makes this system unsuitable for the low-speed operation. In order to minimize the power loss, a symmetrical null-flux topology to reduce the drag force generated by the suspension system was proposed [2]. The Halbach arrays of the null-flux topology are settled with their strong side opposing each other, as shown in Fig. 1(a). The symmetrical null-flux configuration can enhance the horizontal field components while reducing the vertical field components, thus making it possible for a maglev train to mitigate the power loss under at low-speed condition.

A variety of analytical methods have been proposed to analyze the levitation characteristics of the EDS system. In [3], the 2-D force equations for describing a single-sided topology were derived, and the feasibility was verified by experiments. However, a large calculation error of the 2-D model made the analytical approach unreliable. In [4] and [5], an accurate 3-D eddy current field model of the suspension device was established, resulting in the calculation error between analytical and 3-D finite-element method (FEM) being reduced to less than $2 \%$.

Based on the null-flux configuration, a novel asymmetrical double-sided topology of a suspension device is introduced in this paper. The characteristics of the presented system are obtained via the 3-D analytical model. By adjusting the height ratio $(\varepsilon)$ of upper and lower PM arrays, a balance between different optimal objectives of suspension device can be accomplished from the Pareto front by using the dynamic kriging (DK) surrogate model-assisted multiobjective algorithm.

Manuscript received November 3, 2018; revised December 29, 2018; accepted January 18, 2019. Corresponding author: S. Xiao (e-mail: xiaosong@home.swjtu.edu.cn).

Digital Object Identifier 10.1109/TMAG.2019.2894709

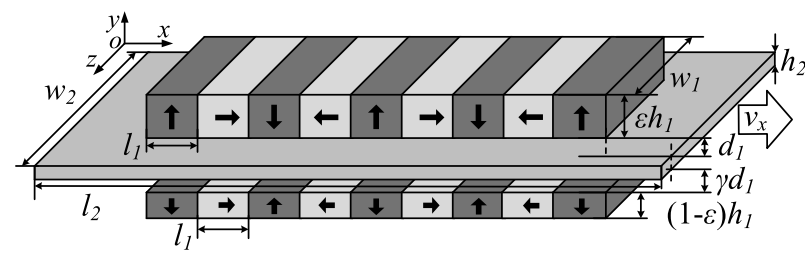

(a)

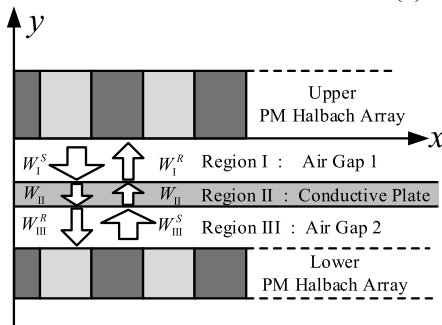

(b)

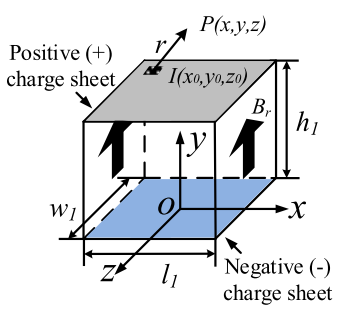

(c)
Fig. 1. (a) 3-D schematic of the double-sided topology. (b) 2-D layer model. (c) Charge model of single magnet.

\section{Analytical Model}

Both the levitation and drag force are generated by the interaction of the source field with the eddy current field; thus, it is necessary to establish analytical models for PM as well as the conductive plate, respectively. For simplification, two assumptions have been made: 1) the plate is large enough to ensure that all the fields are reduced to 0 at the edges and 2) there is no vertical component in eddy current vector.

\section{A. Eddy Current Field}

Since the analytical model of the 3-D eddy current field involves solving three scalar terms as well as the coupling within the magnetic flux density components, the second-order vector potential (SOVP) needs to be introduced

$$
\left\{\begin{array}{l}
\mathbf{B}=\nabla \times \mathbf{A}=\nabla \times(\nabla \times \mathbf{W}) \\
\mathbf{W}=\vec{y} \cdot W
\end{array}\right.
$$

where $\mathbf{B}$ is the flux density, $\mathbf{A}$ is the magnetic vector potential, and $\mathbf{W}$ is the SOVP [5]. The calculation field is divided into 
three regions, which are presented in Fig. 1(b), while the SOVP of each region can be formulated as

$$
\begin{cases}\nabla^{2} W_{\mathrm{I}}^{R}=0 & \text { Region I } \\ \nabla^{2} W_{\mathrm{II}}=-\mu_{0} \sigma \mathbf{v} \cdot\left(\nabla W_{\mathrm{II}}\right) & \text { Region II } \\ \nabla^{2} W_{\mathrm{III}}^{R}=0 & \text { Region III }\end{cases}
$$

where $\sigma$ is the conductivity and $\mathbf{v}$ is the velocity vector. Solving (2) using the separation of variables, SOVPs of the eddy current field on the surfaces of the plate can be derived as

$$
\left\{\begin{array}{l}
W_{\mathrm{I}}^{R}(x, y, z)=\sum_{m=-\infty}^{+\infty} \sum_{n=-\infty}^{+\infty} C_{m n}^{\mathrm{I} R} e^{j \xi_{m} x} e^{j k_{n} z} e^{-\kappa_{m n}\left(y+\sigma_{1}\right)} \\
W_{\mathrm{III}}^{R}(x, y, z)=\sum_{m=-\infty}^{+\infty} \sum_{n=-\infty}^{+\infty} C_{m n}^{\mathrm{III} R} e^{j \xi_{m} x} e^{j k_{n} z} e^{\kappa_{m n}\left(y+\sigma_{1}+h_{2}\right)}
\end{array}\right.
$$

where

$$
\left\{\begin{array}{l}
\xi_{m}=2 \pi m / l_{1}, \quad k_{n}=2 \pi n / w_{1} \\
\kappa_{m n}=\sqrt{\xi_{m}^{2}+k_{n}^{2}}
\end{array}\right.
$$

and the boundary conditions in terms of (2) are

$$
\begin{aligned}
& \left\{\begin{array}{l}
W_{\mathrm{I}}^{S}+W_{\mathrm{I}}^{R}=W_{\mathrm{II}} \\
\frac{\partial W_{\mathrm{I}}^{S}}{\partial y}+\frac{\partial W_{\mathrm{I}}^{R}}{\partial y}=\frac{\partial W_{\mathrm{II}}}{\partial y} \quad \text { at } y=-d_{1}
\end{array}\right. \\
& \left\{\begin{array}{l}
W_{\mathrm{III}}^{S}+W_{\mathrm{III}}^{R}=W_{\mathrm{II}} \\
\frac{\partial W_{\mathrm{III}}^{S}}{\partial y}+\frac{\partial W_{\mathrm{III}}^{R}}{\partial y}=\frac{\partial W_{\mathrm{II}}}{\partial y} \quad \text { at } y=-d_{1}-h_{2} .
\end{array}\right.
\end{aligned}
$$

\section{B. Source Field}

The source field can be viewed as a superposition of all charge sheets. For instance, the 3-D charge model of a single $\mathrm{PM}$ with the positive $y$-direction magnetized can be described by two charge sheets with different properties (positive/ negative magnetic charge sheet), as shown in Fig. 1(c). The equation of the flux density along the normal direction can be expressed as

$$
B_{y}^{\text {Cube }}\left(x, y, z, x_{0}, z_{0}\right)=\frac{B_{r}}{4 \pi} \sum_{i=+,-}\left\{-i \cdot \operatorname{atan}\left[\frac{\left(z-z_{0}\right)\left(x-x_{0}\right)}{\left(y-y_{0}\right) r_{i}}\right]\right\}
$$

where

$$
\begin{aligned}
& r_{+}=\sqrt{\left(x-x_{0}\right)^{2}+\left(y+h_{1}\right)^{2}+\left(z-z_{0}\right)^{2}} \\
& r_{-}=\sqrt{\left(x-x_{0}\right)^{2}+(y)^{2}+\left(z-z_{0}\right)^{2}}
\end{aligned}
$$

and $B_{r}$ is the remanence of PM. Substituting the limits of $x_{0}$ and $z_{0}$ into (7) and (8), the normal field generated by one rectangular PM [Fig. 1(c)] can be written in the form

$$
\begin{aligned}
& B_{y}^{\text {Cube }}(x, y, z) \\
& \quad=\sum_{m=1}^{2} \sum_{n=1}^{2}\left[(-1)^{m+n} B_{y}^{\text {Cube }}\left(x, y, z,(-1)^{m} \frac{l_{1}}{2},(-1)^{n} \frac{w_{1}}{2}\right)\right] .
\end{aligned}
$$

TABLE I

PARAMETERS

\begin{tabular}{llll}
\hline \hline Parameters & Unit & FEM (Initial design) & EXP \\
\hline Length of magnet, $l_{l}$ & $\mathrm{~mm}$ & 120 & 50 \\
Width of magnet, $w_{l}$ & $\mathrm{~mm}$ & 320 & 100 \\
Height of magnet, $h_{l}$ & $\mathrm{~mm}$ & 160 & 50 \\
Length of plate, $l_{2}$ & $\mathrm{~mm}$ & - & - \\
Width of plate, $w_{2}$ & $\mathrm{~mm}$ & 960 & 400 \\
Thickness of plate, $h_{2}$ & $\mathrm{~mm}$ & 5 & 3 \\
Upper air gap, $d_{1}$ & $\mathrm{~mm}$ & 30 & 21 \\
Height ratio, $\varepsilon$ & - & $0.5 / 1$ & 0.5 \\
Air-gap ratio, $\gamma$ & - & 1.5 & 2 \\
Remanence, $B_{r}$ & Tesla & 1.25 & 1.18 \\
Conductivity, $\sigma$ & $\mathrm{S} / \mathrm{m}$ & $2.54 \mathrm{e} 7$ & $2.54 \mathrm{e} 7$ \\
Number of PMs, $N$ & - & 9 & 9 \\
Max Speed, $v_{x}$ & $\mathrm{Km} / \mathrm{h}$ & 180 & 90 \\
\hline \hline
\end{tabular}

The charge model of a randomly magnetized PM can be derived by the coordinate transformation [6]. Therefore, the equation of the source field can be derived by

$$
B_{y}^{S}(x, y, z)=\sum_{\text {All PMs }} B_{y}(x, y, z) .
$$

The SOVP of the source field at the surface of the plate is

$$
\left\{\begin{array}{l}
W_{\mathrm{I}}^{S}=\sum_{m=-\infty}^{+\infty} \sum_{n=-\infty}^{+\infty} \frac{S_{m n}^{\mathrm{IS}}}{\kappa_{m n}^{2}} e^{j \xi_{m} x} e^{j k_{n} z} e^{\kappa_{m n}\left(y+d_{1}\right)} \\
W_{\mathrm{III}}^{S}=\sum_{m=-\infty}^{+\infty} \sum_{n=-\infty}^{+\infty} \frac{S_{m n}^{I I I S}}{\kappa_{m n}^{2}} e^{j \xi_{m} x} e^{j k_{n} z} e^{-\kappa_{m n}\left(y+d_{1}+h_{2}\right)} .
\end{array}\right.
$$

Fourier coefficients $S_{m n}^{I S}$ and $S_{m n}^{\mathrm{IIIS}}$ can be calculated by the Fourier transformation of (10).

\section{Analytical Model of Electromagnetic Force}

By substituting (3) and (11) into the boundary conditions, the undetermined coefficients $C_{m n}^{\mathrm{IS}}$ and $C_{m n}^{\mathrm{IIIS}}$ of the eddy current field can be solved. The electromagnetic tensors of the upper/lower surface of the conductive plate are derived as

$$
\begin{aligned}
\mathbf{F}_{\text {Upper }}= & -\frac{1}{\mu_{0}} \int_{x} \int_{z} \mathbf{B}^{\mathrm{I} R}\left(x,-d_{1}, z\right) \cdot B_{y}^{\mathrm{I} S}\left(x,-d_{1}, z\right) d x d y \\
\mathbf{F}_{\text {Lower }}= & \frac{1}{\mu_{0}} \int_{x} \int_{z} \mathbf{B}^{\mathrm{III} R}\left(x,-d_{1}-h_{2}, z\right) \\
& \cdot B_{y}^{\mathrm{IIIS}}\left(x,-d_{1}-h_{2}, z\right) d x d y .
\end{aligned}
$$

The steady-state [7] force vector is given by

$$
\mathbf{F}=\mathbf{F}_{\text {Upper }}+\mathbf{F}_{\text {Lower }}
$$

\section{Verification}

In order to verify the validity and accuracy of the analytical model, 3-D FEM calculations and measurements using an experimental setup have been conducted [8]; the parameters are shown in Table I.

The comparisons between the analytical model and FEM are shown in Fig. 2, in which (a) and (b) describe the force curves of null-flux $(\varepsilon=0.5)$ and single-sided $(\varepsilon=1)$ suspension devices, respectively. Fig. 3(a) shows the symmetrical null-flux 


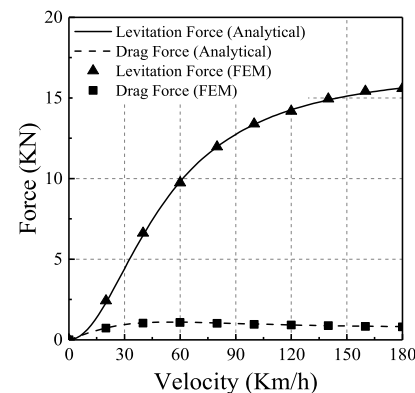

(a)

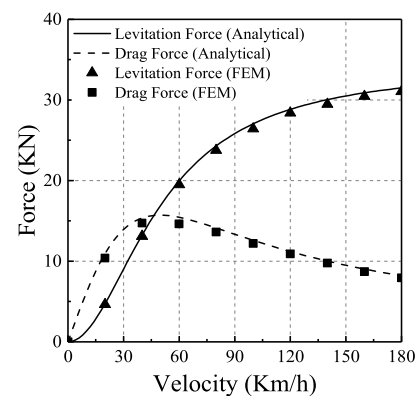

(b)
Fig. 2. (a) Null-flux $(\varepsilon=0.5)$ topology. (b) Single-side $(\varepsilon=1)$ topology.

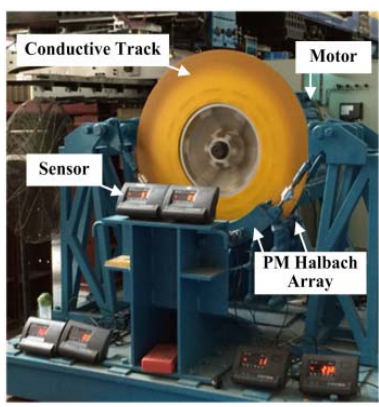

(a)

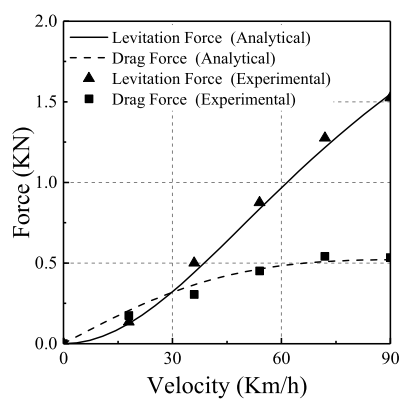

(b)
Fig. 3. (a) Experimental device. (b) Verification result.

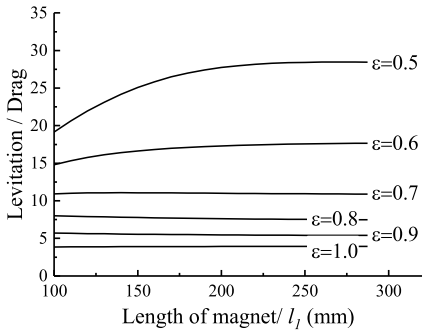

(a)

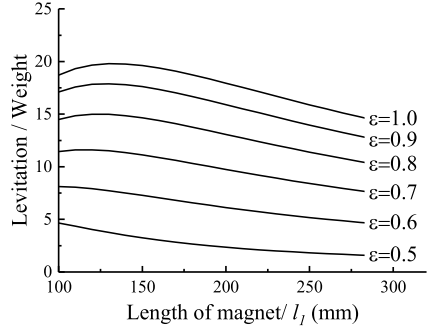

(b)
Fig. 4. Variations of suspension performance with $l_{1}$ for different $\varepsilon$. (a) Levitation/drag ratio. (b) Levitation/weight ratio.

device for experiments. The conductive track is simulated by a circular aluminum plate, while six sensors are utilized to measure the forces. The levitation and drag forces obtained from the analytical method and experiment are shown in Fig. 3(b). The agreement is considered to be very satisfactory.

\section{PARAmeter Analysis}

To observe the performance characteristics of the suspension device with different values of the height ratio $\varepsilon$, parametric sweep analyses were carried out using the analytical model. Figs. 4-7 show the variation of the levitation/drag and levitation/weight ratios when varying one certain structural parameter, while other parameters were fixed as the initial setting of Table I.

Fig. 4(a) shows that the double-sided topology $(\varepsilon<1)$ has a better potential at the levitation/drag ratio following with the increment of $l_{1}$. Fig. 4(b) shows that the optimal performance of levitation/weight ratio exists only when $\varepsilon$ is larger than 0.6.

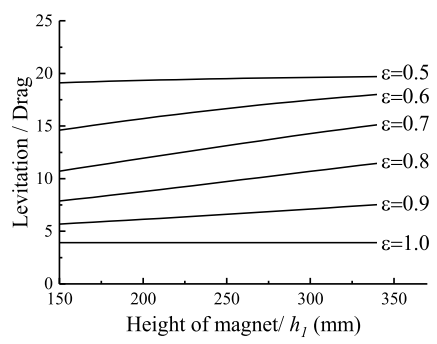

(a)

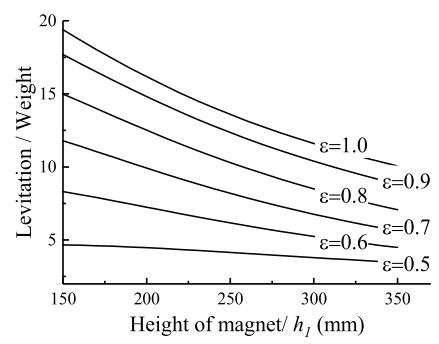

(b)
Fig. 5. Variations of suspension performance with $h_{1}$ for different $\varepsilon$. (a) Levitation/drag ratio. (b) Levitation/weight ratio.

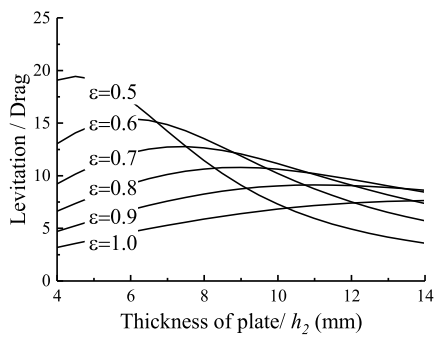

(a)

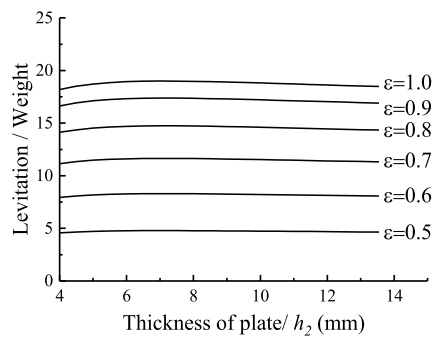

(b)
Fig. 6. Variations of suspension performance with $h_{2}$ for different $\varepsilon$. (a) Levitation/drag ratio. (b) Levitation/weight ratio.

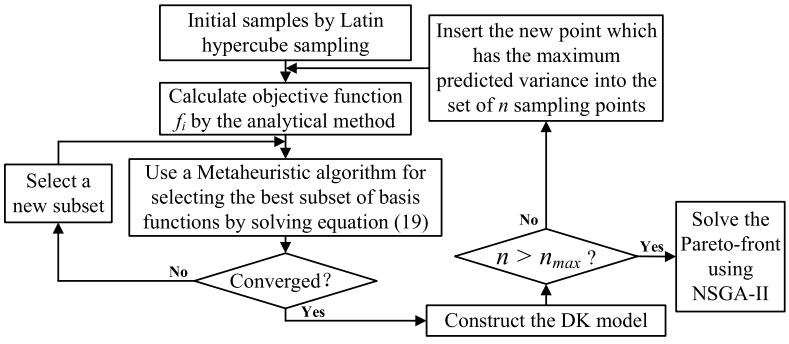

Fig. 7. Procedure of the DK-assisted multi-objective genetic algorithm.

Fig. 5(a) shows that the levitation/drag ratio is improved with the increment of $h_{1}$ while $\varepsilon$ is located within a certain range $(0.6<\varepsilon<0.9)$. For the levitation/drag ratio shown in (b), the thinner magnets result in better performance of the device.

Since all the forces of the suspension device are generated by the interaction of the source field with the eddy current field, the skin effect of the conductive plate must be taken into account. If the thickness of the plate is significantly larger than the skin depth, the drag force produced by a null-flux suspension device will be the superposition of two singlesided ones. Thus, as shown in Fig. 6(a), following with the increment of $h_{2}$, the value of the levitation/drag ratio increases initially, then gradually declines. Finally, Fig. 6(b) shows that the impact of plate's thickness on the levitation/weight ratio is marginal.

\section{Multi-Objective Optimization}

\section{A. Objective Functions}

The design target of the optimization problem has been set to maximize the levitation/drag and levitation/weight ratios; 
TABLE II

DESIGN VARIABLES

\begin{tabular}{llllll}
\hline \hline Parameter & $\varepsilon$ & $l_{1}$ & $h_{1}$ & $h_{2}$ & $\gamma$ \\
Max & 0.5 & $100 \mathrm{~mm}$ & $150 \mathrm{~mm}$ & $4 \mathrm{~mm}$ & 1.2 \\
Min & 1 & $240 \mathrm{~mm}$ & $300 \mathrm{~mm}$ & $14 \mathrm{~mm}$ & 2 \\
\hline \hline
\end{tabular}

thus, the objective functions are defined as

$$
\operatorname{Maximize}\left\{\begin{array}{l}
f_{1}\left(\varepsilon, l_{1}, h_{1}, h_{2}, \gamma\right)=F_{y} / F_{x} \\
f_{2}\left(\varepsilon, l_{1}, h_{1}, h_{2}, \gamma\right)=F_{y} /\left(N \rho l_{1} h_{1} w_{1}\right)
\end{array}\right.
$$

where $\rho$ is the density of PM and $\rho=7.5 \mathrm{~g} / \mathrm{cm}^{3}$.

The vectors of the design variables are shown in (14), and their values are set in Table II; other fixed parameters are set to be the same as for the initial design.

\section{B. Dynamic Kriging-Assisted Multi-Objective Optimization}

The kriging method is an effective way to search for the global optimum with limited given information [9]. The correlation function of the kriging surrogate model consists of a linear regression model and a Gaussian correlation model

$$
Z(x)=\sum_{k=1}^{m} \beta_{k} \boldsymbol{F}^{T}+\varepsilon(x)
$$

where $\boldsymbol{F}$ and $\boldsymbol{\beta}_{\boldsymbol{k}}$ are the vectors of basis functions and regression coefficients, respectively, and $\varepsilon(x)$ is the stochastic process with zero mean and variance $\delta^{2}$. The Gaussian correlation function of $\varepsilon(x)$ may be expressed as

$$
R\left(\varepsilon\left(x^{i}\right), \varepsilon\left(x^{i}\right)\right)=\Pi_{k=1}^{n} e^{-\theta_{k}\left|x_{k}^{i}-x_{k}^{j}\right|^{2}}
$$

where parameter $\theta_{k}$ is the $k$ th process correlation parameter and can be optimized by the maximum likelihood estimator. In general, the form of basis functions $\boldsymbol{F}$ is fixed during the entire modeling process. In this paper, an approach which can dynamically select basis functions is used for constructing the surrogate models, namely, the DK method [10]. All the candidate basis functions are polynomial forms and the full set of $\boldsymbol{F}$ is listed as

$\boldsymbol{F}=\left[1, x_{1}, x_{2} \ldots \ldots x_{k}, x_{1}^{2}, x_{1} x_{2} \ldots \ldots x_{1} x_{k} \ldots \ldots x_{k}^{P}\right]$

where $k$ is the dimension of the variable and $P$ is the highest order of basis functions. They are constrained by

$$
C_{k+P}^{P} \leq n
$$

where $n$ is the number of sampling points.

In order to optimally select the basic functions from (17) so that the surrogate model would have the best accuracy, the sub-optimization problem can be defined as

$$
\text { Minimize } S\left(\mathbf{F}_{\text {opt }}\right)=\frac{1}{N} \sum_{i=1}^{N} s^{2}\left(x_{i}\right)
$$

where $N$ is the number of all the testing points in the design space. $s^{2}\left(x_{i}\right)$ is the predicted variance at $x_{i}$, which is given by $s^{2}(x)=\delta^{2}\left[1-\boldsymbol{r}^{-1} \boldsymbol{R}^{-1} \boldsymbol{r}+\left(\boldsymbol{F}^{T} \boldsymbol{R}^{-1} \boldsymbol{F}\right)^{-1}\left(1-\boldsymbol{F}^{T} \boldsymbol{R}^{-1} \boldsymbol{r}\right)^{2}\right]$

where $\boldsymbol{r}\left(x_{i}\right)$ is the correlation vector between the prediction location and all $N$ samples.

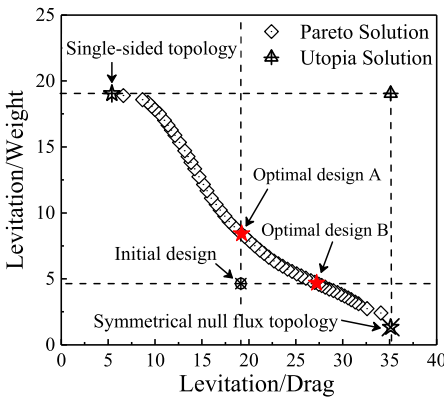

Fig. 8. Pareto front of the multi-objective optimization.

TABLE III

PERFormanCES OF INITIAL AND OPTIMAL DESIGNS

\begin{tabular}{cccccccc}
\hline \hline Parameter & $\varepsilon$ & $l_{I}$ & $h_{1}$ & $h_{2}$ & $\gamma$ & $f_{I}$ & $f_{2}$ \\
Initial & 0.50 & $120 \mathrm{~mm}$ & $160 \mathrm{~mm}$ & $5 \mathrm{~mm}$ & 1.5 & 19.15 & 4.64 \\
Design A & 0.70 & $210 \mathrm{~mm}$ & $240 \mathrm{~mm}$ & $9.4 \mathrm{~mm}$ & 1.32 & 18.74 & 8.64 \\
Design B & 0.60 & $215 \mathrm{~mm}$ & $290 \mathrm{~mm}$ & $6.6 \mathrm{~mm}$ & 1.27 & 26.67 & 4.46 \\
\hline \hline
\end{tabular}

After constructing the DK models of $f_{1}$ and $f_{2}$ defined in (14), the multi-objective optimization is implemented by the non-dominated sorting genetic algorithm based on the final surrogate models. The detailed procedure of the combined algorithm is shown in Fig. 7.

\section{Optimization Results}

In order to construct the dynamic surrogate model for each objective function, first, 100 initial sampling points were randomly generated by the Latin hypercube design [9]. The sub-optimization problem formulated as (19) was solved by the binary particle swarm optimization algorithm. The procedures for the model construction were terminated when the number of sample points exceeded 500. The genetic algorithm was set with the crossover and mutant rates of 0.8 and 0.4 , respectively, 150 individuals, and maximum 100 iterations.

After optimization, the Pareto front is shown in Fig. 8. The extreme cases in the Pareto solutions correspond to two typical topologies of the suspension device, namely, the single-sided topology and symmetrical null-flux topology. Therefore, the weight of performances can be selected in a balanced way via adjusting the height ratio $(\varepsilon)$ of the upper and lower PM arrays.

Two optimal solutions (designs A and B) were selected to be compared with the initial design. The design parameters and performances of those three cases are shown in Table III. In optimal design A, the levitation/drag ratio is 18.74 , which is approximately the same as the initial case (19.15), the levitation/weight ratio is 8.64, almost twice of the initial case (4.64). Compared with design A, design B has a better performance at the levitation/drag ratio (26.67) than initial design, while the levitation/drag ratio is similar. Generally, all the optimal configurations in the Pareto front between designs $\mathrm{A}$ and $\mathrm{B}$ are superior to the initial design in two objectives. An outstanding improvement can be achieved while the asymmetrical topology is adopted in the EDS device.

\section{CONCLUSION}

A methodology is proposed for the analysis and optimal design of a novel asymmetrical EDS device. This has allowed 
overcoming the extreme performances of the conventional EDS configuration. The characteristics of the device are established and illustrated by conducting a parameter sweep based on the SOVP-derived analytical model. Moreover, a multiobjective optimization problem was defined and carried out by a hybrid algorithm to find the balance between the two optimal objectives of EDS devices. The Pareto front shows that the proposed device outperforms conventional topologies in terms of both the levitation-drag and levitation-weight ratios compared with the original design.

\section{ACKNOWLEDGMENT}

This work was supported by the National Natural Science Foundation of China under Grant 51707166.

\section{REFERENCES}

[1] D. B. Montgomery, "Overview of the 2004 magplane design," in Proc. 18th Int. Conf. Magnetically Levitated Syst. Linear Drives, Shanghai, China, 2004, pp. 106-113.

[2] R. Kratz and R. F. Post, "A null-current electro-dynamic levitation system," IEEE Trans. Appl. Supercond., vol. 12, no. 1, pp. 930-932, Mar. 2002.
[3] H.-W. Cho, H.-S. Han, J.-S. Bang, H.-K. Sung, and B.-H. Kim, "Characteristic analysis of electrodynamic suspension device with permanent magnet Halbach array," J. Appl. Phys., vol. 105, no. 1, 2009, Art. no. 07A314.

[4] Y. Chen, W. Zhang, J. Z. Bird, S. Paul, and K. Zhang, "A 3-D analytic-based model of a null-flux Halbach array electrodynamic suspension device," IEEE Trans. Magn., vol. 51, no. 11, Nov. 2015, Art. no. 8300405 .

[5] S. Paul, W. Bomela, N. Paudel, and J. Z. Bird, "3-D eddy current torque modeling," IEEE Trans. Magn., vol. 50, no. 2, pp. 905-908, Feb. 2014.

[6] J. Duan, S. Xiao, K. Zhang, and Y. Jing, "A novel 3-D analytical modeling method of trapezoidal shape permanent magnet Halbach array for multi-objective optimization," J. Elect. Eng. Technol., vol. 14, no. 2, pp. 635-643, Mar. 2019.

[7] I. Moeini, M. Ahmadpour, A. Mosavi, N. Alharbi, and N. E. Gorji, "Modeling the time-dependent characteristics of perovskite solar cells," Sol. Energy, vol. 170, pp. 969-973, Aug. 2018.

[8] M. Houshmand, M. H. Zandi, and N. E. Gorji, "Simulation analysis of thermal degradation in graphene back contacted CdTe ultrathin films," IEEE Trans. Nanotechnol., vol. 14, no. 3, pp. 493-496, May 2015.

[9] S. Xiao, M. Rotaru, and J. K. Sykulski, "Adaptive weighted expected improvement with rewards approach in Kriging assisted electromagnetic design," IEEE Trans. Magn., vol. 49, no. 5, pp. 2057-2060, May 2013.

[10] B. Xia, N. Baatar, Z. Ren, and C.-S. Koh, "A numerically efficient multi-objective optimization algorithm: Combination of dynamic Taylor Kriging and differential evolution," IEEE Trans. Magn., vol. 51, no. 3, Mar. 2015, Art. no. 9400604. 